\title{
La presse satirique face aux assassinats politiques au Togo en 1992 : quel crédit pour l'historien ? Les exemples de $L a$ parole et Kpakpa désenchanté
}

\begin{abstract}
A imprensa satírica em torno dos assassinatos políticos no Togo em 1992: que crédito para o historiador? Exemplos de La parole e Kpakpa désenchanté
\end{abstract}

The satirical press against the political assassinations in Togo in 1992: What scope for the historian? Examples of La parole and Kpakpa désenchanté

Koffi Nutefé Tsigbe*

Résumé: Au sortir de la Conférence nationale dite souveraine en 1991, le Togo fut le théâtre d'une succession de violences politiques. L'une des caractéristiques de ce mouvement fut les assassinats politiques organisés en 1992. En l'absence de sources d'archives pouvant permettre d'étudier cette question à l'aune de l'histoire immédiate, le présent article questionne deux organes de la presse satirique de l'époque (La parole et Kpakpa désenchanté) et décrit le contexte, les acteurs et les modes d'opération desdits assassinats politiques. Par ailleurs, il fait une extrapolation pour expliquer comment l'historien peut, en l'absence d'archives, avoir recours à ce type de sources et comment le traiter pour écrire l'histoire.

Mots-clés: Togo, presse, satire, caricature, assassinat politique.

Resumo: Ao final da conferência nacional, chamada soberana, em 1991, o Togo foi cenário de uma sucessão de atos de violência política. Característicos dessa violência foram os assassinatos políticos em 1992. Na ausência de quaisquer

(c) EY Direito autoral e licença de uso: Este artigo está licenciado sob uma Licença Creative Commons. Com essa licença você pode compartilhar, adaptar, para qualquer fim, desde que atribua a autoria da obra, forneça um link para a licença, e indicar se foram feitas alterações. 
fontes de arquivos que permitam estudar esta questão à luz da história imediata, o presente artigo aborda a imprensa satírica daquele ano (La parole e Kpakpa désenchanté) e mostra o contexto, os atores e os modos de operação dos ditos assassinatos políticos. Tenta-se ainda explicar como o historiador pode, na ausência dos arquivos, recorrer a esse tipo de fontes e como tratá-lo para escrever a história.

Palavras-chave: Togo, imprensa, sátira, caricatura, assassinato político.

\begin{abstract}
At the end of the national Conference in 1991, Togo registereda succession of political violence. One of the characteristics of this political violence is political killings organized in 1992. In the absence of archival sources to study this issue in the light of immediate history, this article questions the satirical press of that time (La parole and Kpakpa désenchanté) and shows the context, actors and the modes of operation of those political killings. In addition, he extrapolates to explain how the historian can, in the absence of the archives, have recourse to this type of sources and how to treat it to write the history.
\end{abstract}

Keywords: Togo, press, satire, caricature, political assassination.

\title{
Introduction
}

Dans un précédent article paru en octobre 2013 dans le $\mathrm{n}^{\circ} 015$ de la revue « Mosaïque $»,{ }^{1}$ nous avons montré que, quand bien même image et histoire sont un couple qui n'a pas toujours fait bon ménage, les données iconographiques, traitées selon la méthode critique héritée de l'École historique méthodique, sont d'excellentes sources pouvant permettre l'écriture de l'histoire du Togo indépendant en l'absence des archives. Ce même article fait référence à la caricature qui, traitée rigoureusement, peut permettre de saisir une bonne trame de l'histoire immédiate de ce pays. La présente contribution s'inscrit dans cette logique. Elle s'appuie sur deux journaux satiriques pour décrypter l'histoire des violences, notamment les assassinats politiques au Togo au cours de l'année 1992.

En effet, le processus démocratique entamé au Togo le 5 octobre 1990 a donné lieu, non seulement à la liberté d'expression mais aussi à des dérapages ayant occasionné des violences politiques. La presse satirique qui, entre temps, a émergé, a eu pour cible la vie politique togolaise, précisément, les assassinats politiques, à un moment où le pont fut rompu entre l'opposition dite démocratique et les partisans du Rassemblement du peuple togolais (RPT), hier parti unique, fragilisé dans les sillages dudit processus démocratique. Les hebdomadaires La Parole et Kpakpa désenchanté, émergés tous deux en 1991, 
ayant paru pendant deux ans pour le premier et quatre ans pour le second, ont fait de ces assassinats politiques, leur sujet de prédilection. Quelle lecture ces deux organes ont-ils fait de ces malheureux événements connus au Togo au cours de l'année 1992? Quelle est l'origine de ces journaux ? Quelle fut leur ligne éditoriale ? Comment ont-ils présenté ces faits dans leurs parutions de 1992 ? Telles sont les questions qui retiennent notre attention dans la présente contribution.

Le choix de 1992 se justifie par le fait que c'est la seule année au cours de laquelle les deux journaux satiriques ont publié ensemble des séries complètes. Avant, ce fut La parole seule qui était dans les kiosques jusqu'en octobre 1991. À partir de cette date, les deux ont paru ensemble, mais les 12 premiers numéros de Kpakpa désenchanté se rapportant à l'année 1991 n'ont pas été retrouvés à la Bibliothèque nationale togolaise (BNT) où ils sont censés être archivés. Après 1992, c'est Kpakpa désenchanté qui fit cavalier seul. C'est donc le souci de comparaison qui a guidé ce choix.

Par presse satirique, il est entendu les journaux qui, par écrit ou par dessin, s'attaquent aux vices et aux ridicules de la période en rapport avec les violences politiques. Elle inclut la caricature qui, selon le Dictionnaire des termes artistiques de Filippo Baldinucci,

correspond chez les peintres et les sculpteurs à une méthode consistant à faire un portrait le plus ressemblant possible, mais aussi à augmenter et à accentuer exagérément les défauts des traits qu'ils copient. Ainsi, le portrait, dans son ensemble, est parfaitement conforme au modèle, alors que les traits qui les composent sont modifiés. ${ }^{2}$

Associée à l'humour et à la critique, la caricature donne également au caricaturiste la liberté d'amplifier les réalités qu'il décrit. Celles-ci concernent, dans le cas d'espèce, les massacres ainsi que l'utilisation de la violence sous toutes ses formes en relation avec la vie politique togolaise d'alors.

Si en Occident, l'utilisation des images ${ }^{3}$ ou de la caricature ${ }^{4}$ a donné lieu à des publications historiques pertinentes, au Togo, en dehors de l'article cité en introduction et de quelques ouvrages ayant utilisé la photographie à titre illustratif, ${ }^{5}$ il est rare de trouver des travaux scientifiques en histoire s'intéresser à cette thématique. En conséquence, la méthodologie adoptée pour traiter ce sujet s'appuie essentiellement sur les deux journaux. Toutefois, mise à part l'analyse de contenu, les éléments de la critique proviennent de tout autre document primaire ou secondaire, portant sur la période d'étude.

Les résultats de l'analyse sont exposés suivant un plan binaire. La première partie s'intéresse à l'émergence de la presse satirique au Togo et présente ensuite les journaux à l'étude. La seconde, plus dense que la 
première, analyse la présentation factuelle et imagée faite par ces journaux de l'assassinat politique et montre les réalités y relatives.

\section{1. Émergence et présentation des deux journaux satiriques au Togo}

$\mathrm{Au}$ Togo, la presse satirique n'a pas émergé sans difficultés. Son émergence a été rendue possible grâce aux luttes menées pour la démocratisation de ce pays.

\subsection{Contexte d'émergence de la presse satirique}

Une lecture minutieuse des travaux sur le couple image/caricature et histoire montre que dans les pays occidentaux par exemple, c'est sous la dictature que la caricature s'est amplement développée. En revanche, au Togo, elle n'a émergé que dans le sillage du processus de démocratisation, lancé le 5 octobre 1990. Avant cette période, les militaires au pouvoir, depuis le deuxième coup d'État du 13 janvier 1967, ont instauré un régime de répression ayant tenu en laisse les Togolais pendant plus de deux décennies. Sous ce régime dictatorial, la liberté d'expression a fortement souffert. Pour le Rassemblement du peuple Togolais (RPT, parti unique pendant cette période), la presse n'avait aucun autre rôle à jouer que d'être un moyen de propagande au bénéfice du chef de l'État. En conséquence, il a été procédé à la confiscation de la liberté des organes de presse et de celle des journalistes. La Commission de la Propagande, de la Presse et de l'Animation du RPT était l'organe de régulation des journaux. De ce fait, seul le parti unique pouvait créer un organe de presse ${ }^{6}$. Dans ce contexte, la presse satirique, humoristique, voire critique et irrévérencieuse n'avait aucune chance de s'exprimer, sans attirer les foudres des thuriféraires sur elle, sous un régime où les contestataires sont considérés comme des dangers publics et une écharde à la " prétendue » unité nationale.

Mais à partir de la décennie 1990, les mutations intervenues dans la politique internationale, occasionnées par l'effondrement du communisme, eurent pour conséquences en Afrique en général, un renouveau dans les systèmes politiques. Ceux-ci se caractérisent, entre autres, par la fin des partis uniques et l'ouverture du continent au multipartisme. La lutte pour la démocratie ainsi lancée a conduit, dans bien des endroits sur le continent, à l'organisation de conférences nationales. Au Togo, elle eut lieu du 8 juillet au 28 août 1991. L'un des acquis de cette conférence est la naissance, à foison, des organes de presse et, plus tard, l'adoption d'une loi plus libérale en $1998^{7}$ en faveur des journaux. Toutefois, avant même que cette loi qualifiée de plus libérale ne soit adoptée, la presse contestataire et satirique émergea. C'est dans ce sillage que la caricature prit ses assises. Les journaux La Parole et Kpakpa désenchanté se sont illustrés dans la satire. 
1.2. La Parole et Kpakpa désenchanté : deux organes satiriques caractéristiques de la liberté d'expression acquise dans les années 1990

\subsubsection{La Parole}

«FOLY Kangni Bertin, né le 5 septembre 1962 à Brazzaville (Congo), fils de Foly Efoé Joseph et de HOFFER Joséphine, Togolais père de deux enfants, Directeur de Publication de l'hebdomadaire «La PAROLE» demeurant à Djidjolé, B.P. 62210 Tél. 21-46-00 [...]». C'est par cette phrase tirée de la citation à comparaître, datée du 11 septembre 1992, adressée par le Procureur de la République Togolaise au nommé Foly Kangni Bertin, publiée par $L a$ Parole dans sa parution $n^{\circ} 83$ du 16 septembre 1992, que l'on a découvert l'identité du Directeur de publication de ce journal.

En effet, comme il était presque de coutume dans le rang des journaux contestataires de l'époque, les parutions de La Parole ne portaient aucune indication pouvant permettre d'identifier le Directeur de publication. De même, les articles étaient presque toujours signés sous des pseudonymes. Cela peut s'expliquer par les réalités de l'époque où les journalistes critiques vis-à-vis du pouvoir, étant dans le viseur des forces de l'ordre, faisaient objet d'agressions physiques. Ainsi, pour ne pas se jeter en pâture à la garde prétorienne, beaucoup d'entre eux ont choisi de ne pas dévoiler leur identité dans leurs parutions. Mais cela n'a pas empêché les pouvoirs publics d'avoir des informations précises sur ces Directeurs de publication comme c'est le cas de Foly Kangni Bertin. Qu'en est-il de l'historique de La Parole?

Il est difficile de répondre de façon exhaustive à cette question car le journal lui-même ne donne aucun indice y afférent. Non seulement nous ne connaissons pas avec précision la date de parution du premier numéro, mais aussi il est en outre difficile d'avancer un chiffre par rapport au nombre total de numéros parus. À la BNT où nous avons effectué nos recherches, nous n'avons trouvé que 24 numéros disponibles pour l'année 1991 et 21 pour 1992. Ce qui est connu avec précision par contre, c'est le nombre d'années au cours desquelles le journal a paru : deux ans (1991-1992). On sait aussi qu'il s'agit d'un hebdomadaire togolais d'information, paraissant tous les mercredis, vendu au Togo au prix de 200 FCFA, au Bénin, 250 FCFA, en Afrique Occidentale, 350 FCFA, en France, 9 FF et dans les autres pays, 12 FF. $^{8}$

Le premier numéro de l'an 1991 disponible à la BNT porte le $\mathrm{n}^{\circ} 10$. Il date du 17 avril. A partir de ces indications, on peut postuler que 9 numéros ont paru avant celui du 17 avril. Considérant que cet organe paraissait une fois par semaine, on peut postuler que le premier numéro serait publié entre le 14 et le 15 février 1991. La dernière parution trouvée à la BNT porte le $\mathrm{n}^{\circ} 91 \mathrm{du}$ 11 novembre 1992. Mais il n'est pas certain que ce soit seulement 91 numéros que La Parole ait publiés durant son existence. Mais faute de données, on ne saurait dire avec précision ce qu'il en est. 
Toute la centaine de numéros répertoriée (par estimation) n'est pas prise en compte dans la présente analyse. Comme déjà mentionné, nous nous sommes intéressé aux 21 numéros disponibles de l'année 1992. Sur les 21, 7 numéros seulement sont retenus, parce que traitant directement de la problématique du sujet qui nous intéresse. Il s'agit des numéros 47 (du $1^{\text {er }}$ janvier 1992), 71 (du 24 juin), 79 (du 19 août), 80 (du 26 août), 83 (du 16 septembre), 87 (du 14 octobre) et 91 (du 11 novembre).

Il faut préciser qu'en dehors de la thématique des assassinats politiques, La Parole développe d'autres sujets tels le plasticage, le tabassage, les menaces de tous genres, la politique générale du Togo, les activités des partis politiques, le détournement des deniers publics et l'impunité, les réalités du monde judiciaire, les relations entre le président togolais et ses collègues africains, les problèmes de cohabitation entre les peuples du Togo, les élections, etc. Il s'agit donc d'un organe très riche en informations, même si les contemporains doutaient parfois de la véracité de certaines données véhiculées dans ses colonnes. Les réalités relatives à Kpakpa désenchanté ne sont pas très différentes de celles qui viennent d'être décrites.

\subsubsection{Kpakpa désenchanté}

Cet hebdomadaire dont le nom signifie littéralement le canard désenchanté, serait né vers octobre 1991. Comme pour le précédent, les informations manquent pour dire avec précision la date de sa création. On sait néanmoins qu'il paraissait chaque mardi et que le tout premier numéro de la série retrouvé à la Bibliothèque nationale du Togo porte le $\mathrm{n}^{\circ} 13$ et date du 7 janvier 1992. Sur la base de ces éléments, les calculs font remonter la parution du $1^{\text {er }}$ numéro à la date du 24 octobre 1991. Malgré l'exactitude des calculs, cette date ne peut qu'être indicative. Il était vendu au Togo au prix de 200 FCFA, au Bénin, 250 FCFA, en Afrique Occidentale, 300 FCFA (contre 350 FCFA pour La Parole), en France, 8 FF (contre 9 FF pour La Parole) et dans les autres pays, $12 \mathrm{FF}^{9}$. Son Directeur de publication s'appelle Knock Kalao Billy.

À la BNT, seuls 118 numéros sont disponibles, à raison de 34 numéros pour l'année 1992, 31 pour 1993 et 53 pour 1994. Mais à vrai dire, les parutions de l'hebdomadaire se sont poursuivies sans doute au moins jusqu'en 1995, puisque de façon fortuite, nous avons découvert un numéro daté du 8 août 1995 portant $n^{\circ} 192$. Encore une fois, il est difficile de dire combien de numéros ont été publiés et quelle a été la durée de vie exacte de cet hebdomadaire. Cependant, des 118 numéros disponibles à la BNT, seuls 14 ont été retenus dans le cadre de notre travail. Il s'agit des numéros 13 (du 07 janvier), 15 (du 21 janvier), 16 (du 4 février), 17 (du 11 février), 22 (du 17 mars), 26 (du 14 avril), 28 (du 28 avril), 30 (du 12 mai), 34 (du 9 juin), 36 (du 23 juin), 41 (du 28 juillet), 45 (du 25 août), 46 (du $1^{\text {er }}$ septembre) et 52 (du 13 octobre). 
Tout comme pour La Parole, on peut lire dans les colonnes de Kpakpa désenchanté, en dehors des questions relatives aux massacres politiques, des sujets relatifs au plasticage, au tabassage, aux menaces de tous genres, aux questions de société (infidélité dans les couples, prostitution, inondations, famine, les jeux de hasard, les mouvements syndicaux, etc.), de politique intérieure (réconciliation armée-nation, célébration des fêtes nationales), de la publicité, ainsi que des informations sur des pays voisins du Togo.

Cette présentation succincte des deux organes montre des problèmes d'archivage auxquels fait face la BNT. Dans le cas d'espèce, soit les organes eux-mêmes n'ont pas été assidus dans le dépôt des exemplaires à la BNT, soit les numéros manquants ont disparu des rayons par manque de vigilance des bibliothécaires. Cette situation ne peut que limiter les chercheurs. Par ailleurs, on se rend compte que les deux organes ont des similitudes : hebdomadaire chacun, satire comme centre d'intérêt majeur, ouverture sur des sujets de société entre autres, prix de vente des numéros presque identiques, majeure partie des articles signée de pseudonymes, presse dans la mouvance de l'opposition au régime, etc. Voilà autant de raisons qui justifientle choix de ces deux organes dans la présente étude. Au-delà de ces ressemblances, il ne faut pas occulter les dissimilitudes entre ces deux hebdomadaires, dont la principale est la non convergence des informations véhiculées. En effet, sur la trentaine de titres recensés (une dizaine pour La Parole et une vingtaine pour Kpakpa désenchanté), c'est à peine si l'on retrouve quatre traités ensemble par les deux organes. Cette situation a certes l'avantage de mettre à la disposition du chercheur, une pluralité d'informations ; mais du point de vue méthodologique, elle ne lui donne pas assez de marge pour opérer la critique. Toutefois, étant averti, le chercheur se donnera d'autres moyens de critique pour donner un sens à l'analyse. Malgré tout, les informations publiées convergent vers une réalité : l'année 1992 a été témoin de violences politiques de tous ordres au Togo.

\section{Le contexte de l'année 1992 et les assassinats politiques la caractérisant : quel intérêt pour l'historien ?}

On ne peut comprendre les massacres politiques relatés par les deux organes à l'étude en 1992 qu'en les inscrivant dans leur contexte.

\subsection{Le contexte de l'année 1992 au Togo}

De sources concordantes, l'année 1992 a été caractérisée par des violences politiques. Que ce soit Tété-Adjalogo, ${ }^{10}$ Kondi Charles Madjome Agba ${ }^{11}$ le Parti des travailleurs ${ }^{12}$ ou encore Essohanam Batchana, ${ }^{13}$ Kodjo Koffi ${ }^{14}$ chacun de ces auteurs montre, à sa manière, ce qui a rendu cette année violente.

En effet, les travaux de la Conférence nationale ont débouché, le 26 août 1991, sur l'élection d'un premier ministre du nom de Joseph Kokou Koffigoh 
et de 70 délégués au Haut conseil de la République, parlement de transition présidé par le prélat Mgr Philippe Fanoko Kpodzro. ${ }^{15}$ Ayant pour mission de conduire une période transitoire au terme de laquelle devront être organisées des élections présidentielles, le premier ministre a formé un gouvernement dit de transition. Ce dernier a connu une vie éphémère, puisque le 3 décembre 1991, un coup de force militaire a tenté de le renverser. ${ }^{16}$ Cette situation fut l'une des causes de la violence politique de 1992. Selon Kondi Charles Madjome Agba : «L'année 1992 reste [...] celle de la généralisation -et de la banalisation- de la violence politique : violence verbale, manifestations de rues, affrontements interethniques, attentats, meurtres, plasticages de maisons. L'insécurité est partout $\gg{ }^{17}$ Les raisons de cette insécurité sont multiples. On peut citer, entre autres, les séries de marches de protestations des populations à travers le pays pour dénoncer l'intrusion de l'armée dans la vie politique sous le gouvernement de transition; en riposte, les forces armées ont fait des représailles contre, d'une part, les populations à la base et d'autre part, les institutions, notamment le Haut conseil de la République dont les membres en étaient la cible. ${ }^{18} \mathrm{Il}$ s'est alors installé une crise de confiance entre les militaires et les civils. C'est bien cette crise qui a rendu les relations tendues entre les deux camps, occasionnant des violences politiques au cours de cette année 1992.

Ce contexte de l'année 1992 a été bien présenté par les deux journaux. En effet, dans ses vœux aux Togolais, Kpakpa désenchanté écrit : « Kpakpa vous souhaite pour 92, dans le hiahiahia, sans chuan, ses vœux de bonbon et lalaleureuse année $\gg .{ }^{19}$ Cette formule alambiquée voudrait dire que le journal espère que l'année se déroulera dans la joie sans série de malheurs. Mais en le déclarant, le journal lui-même était quelque peu dubitatif, puisque juste en dessous de ces vœux, il titre : " Le début d'un festival d'explosions en 92 : Bombe année à Sokodé ». Dans le développement, il dit en substance :

[...] Nous vous l'avons «souhaitée», cette «BOMBE ANNÉE». Les petits esprits ont cru à une plaisanterie, oubliant scandaleusement que notre Abalo national détient toutes les caractéristiques pour être classé parmi les tout premiers hommes de la terre. [...] Sachez tout simplement pauvres con-patriotes que cette année, l'INSECURITÉ nous est garantie à $200 \%$. [... . ${ }^{20}$

Il poursuit en citant une série d'attentats orchestrés contre les leaders de l'opposition dite démocratique, qui se sont succédé entre le 4 et le 6 janvier, présageant d'une année " affreuse ». Avant de conclure, le journal avertit : « Des coups de bombe pareils sont programmés pour toute la saison et visent les journaux privés, les chefs de partis, les responsables d'associations des villes et villages $[\ldots]$, tant que Gnass sera là en chair et en awooyOS $» .^{21}$ 
Pour le journal, celui qui garantit l'insécurité pour l'année 1992, c'est le «Abalo national », c'est-à-dire le président de la République, Gnassingbé Eyadéma, à qui on a attribué le nom Abalo, signifiant en Kabiyè, " garçon », une façon de désigner ses origines. Il est encore appelé « Gnass », diminutif de son nom « Gnassingbé ».

Pour n'avoir pas voulu croire en ses mises en garde prémonitoires au sujet de l'insécurité qui règnera au cours de l'année, les citoyens ont été considérés comme des « cons » dans le jeu de mots «pauvres con-patriotes ». De même, l'existence du président est tournée en dérision, considérant qu'aussi longtemps qu'il se livrera aux beuveries, il garantira toujours l'insécurité aux Togolais. C'est ce que traduit cette portion : « tant que Gnass sera là en chair et en awooyOS ». En lieu et place de l'expression « en chair et en os », c'est écrit « en chair et en awooyOS », Awooyo étant le nom d'une bière locale, la préférée du Président.

La Parole, quand à lui, a mis en garde contre tout ce qui se préparait pour l'année, dans sa parution du $1^{\mathrm{er}}$ janvier 1992. Il cite, entre autres, le HCR avec lequel le Rassemblement du peuple togolais (RPT- parti du Président de la République dont la dissolution a fait l'objet de débats au HCR) voudra en découdre, en projetant l'élimination de son président, le prélat Kpodzro, devenu visiblement gênant. Il a également condamné la proposition du premier ministre de la transition selon laquelle « Pour favoriser [le] processus de réconciliation nationale, il importe qu'une nouvelle loi d'amnistie puisse élargir la portée de celle du 12 avril 1991, compte tenu de la nouvelle donne de la politique nationale $» .^{22}$ Le journal estime qu'appliquer une telle décision, c'est donner carte blanche à la violence politique qui risque de faire partie du quotidien des Togolais en cette année 1992, surtout que les hommes politiques de la transition sont déjà dans le viseur de " l'Air Pété », déformation de la dénomination du parti RPT, montrant la façon dont le journal le dédaigne. Il conclut en ces termes : « Décidément au Togo, pour se faire amnistier, il faut commencer par tuer le maximum $»{ }^{23}$

En définitive, les deux journaux ainsi que les auteurs ayant consacré leurs productions à cette période s'accordent à reconnaître que l'année 1992 n'était pas des plus paisibles pour les Togolais. Que ce soit de façon prémonitoire ou dans la réalité des faits, la littérature existante fait observer sans ambages que l'insécurité était omniprésente, alliant actes de vandalisme et homicides volontaires, surtout orchestrés par les forces de l'ordre. Constamment accusés, ces dernières se défendaient, arguant n'avoir agi par moment qu'en représailles aux provocations multiples des civils. Mais on sait également que leurs différentes actions étaient dictées par la volonté de terroriser les forces de la transition, les poussant à bout pour qu'elles lâchent prise afin de rétablir l'autorité du général Eyadéma mise à mal par la conférence nationale. ${ }^{24}$ Les actes de violences politiques issus des 
relations tendues entre les forces de l'ordre et les civils ont été relatés par les hebdomadaires à l'étude.

2.2. Illustration des assassinats politiques par les deux journaux : quelle portée?

Dans cette rubrique, il sera présenté la chronique des assassinats politiques avant de se pencher sur le crédit que l'historien peut accorder aux informations véhiculées par la satire.

\subsubsection{Chronique des assassinats politiques vus par les journaux}

Une lecture attentive des numéros des deux journaux retenus pour analyse met en lumière les acteurs et les victimes des violences politiques de l'année 1992. Les premiers sont majoritairement les militaires et, accessoirement des miliciens du régime RPT, recrutés, armés et entrainés par les militaires, dans le but de régler des comptes à ceux qui, à tort ou à raison, étaient considérés comme des personae non gratta. S'agissant des victimes, elles sont de plusieurs ordres : les hommes politiques de l'opposition, notamment les membres du HCR, les responsables de partis politiques, les membres du gouvernement de la transition, etc. En dehors de cette catégorie de victimes, on peut citer des honnêtes citoyens (considérés comme supportant ces hommes politiques), des militaires soupçonnés d'être de mèche avec l'opposition, des miliciens du RPT accusés de trahison, les journalistes de la presse privée et même le personnel du monde diplomatique.

Kpakpa désenchanté, dans sa parution du 4 février 1992 a donné le ton. Sous le titre "Adétikopé : les FAT ${ }^{25}$ accusées, la $\mathrm{CNDH}^{26}$ insultée et Koffigoh se tait ? », le journal fait état d'un massacre de quatre jeunes à Adétikopé (banlieue nord de Lomé) orchestré par des militaires togolais. Nommés respectivement Rocco Matthia, Épiphane Dogbévi, Brother de Souza, Kokouvi de Souza, ces jeunes, membres du RPT, auraient été bernés par un autre membre du parti qui leur aurait demandé de l'aider à accomplir une mission relative aux échéances électorales qui s'approchaient, afin de leur permettre de gagner quelques pécules supplémentaires. Mais en réalité, cet homme serait recruté par quelques officiers de l'armée togolaise pour leur donner un coup de main afin d'éliminer des jeunes. C'est dans l'accomplissement de cette "prétendue mission » que ces jeunes furent fusillés la nuit du vendredi 24 janvier 1992 entre 23 heures et 00 heure à Adétikopé. Trois seraient tombés tués sur-le-champ. Kokouvi de Souza, le quatrième, se serait jeté sur le sol pour faire le mort. Rescapé, il fut celui par qui l'information aurait filtré. ${ }^{27}$

Informés de l'affaire, les membres de la CNDH se rendirent sur les lieux le lendemain et, après investigation, accusèrent les FAT. Leur rapport d'investigation fut relayé par la Radio France internationale (RFI), le jeudi 30 
janvier dans le journal du soir. Panique au sein de l'armée, qui démentit les faits, accusant la CNDH d'avoir subtilisé des corps à la morgue de Lomé et déposé sur le site d'Adétikopé, dans le seul but de ternir l'image de l'armée togolaise. ${ }^{28}$ Dans cette affaire, ce qui a le plus choqué, selon le journal, c'est le silence du premier ministre de la transition, Joseph Koffigoh.

Le numéro suivant de l'hebdomadaire est revenu sur les faits. Et c'est là qu'on comprend réellement les dessous de cette affaire. En effet, les jeunes gens assassinés feraient partie d'un groupe au service du Rassemblement du peuple togolais (RPT), ayant pour mission de recruter des jeunes de Lomé pour le parti. Un adhérent inscrit percevait 15000 FCFA alors que le recruteur en percevait le double. Leur activité secondaire consistait à appuyer les FAT à exécuter des casses, des vols à main armée pour maintenir la pression et la panique sur la population qui, selon l'armée, cautionnait les opposants dans leur désir de contraindre le général Eyadéma à la démission de la magistrature suprême. Mais ces jeunes ont commis l'erreur d'avoir déclaré plus d'une fois que, bien qu'étant membres du RPT, ils ne voteraient pas pour son candidat. Ils furent donc assassinés en représailles. ${ }^{29}$ Cette version des faits, a été quelque peu confirmée dans le rapport de la CNDH de l'année en question.

On se rend alors à l'évidence que les victimes des assassinats politiques à l'époque peuvent également être ceux considérés comme des traitres. Ce point de vue est par ailleurs confirmé par l'aventure du capitaine Pello à Kara.

En effet, accusé de « collaborationnisme » avec l'opposition, cet officier de l'armée togolaise, censé être du côté du RPT, a été « attiré dans un traquenard par un groupe d'officiers supérieurs de notre armée $»,{ }^{30}$ qui l'aurait condamné à mort le 26 juillet 1992. L'ayant cherché en vain, ses parents étaient dans l'obligation de lui faire son deuil.

Les hommes politiques surtout de l'opposition, étaient la principale cible des auteurs de cette violence. Nous évoquerons ici deux cas pour illustrer cette situation. Il s'agit de l'attentat de Soudou contre Gilchrist Olympio et l'assassinat de Tavio Amorin à Tokoin Gbonvié.

Dans sa parution du 12 mai 1992, Kpakpa désenchanté titre : «Et si Gigi était mort? ». Il décrit comment, au début de mai 1992, lorsque Gilchrist Olympio, président de l'Union des forces de changement (UFC), organisa une tournée à l'intérieur du pays pour y implanter des sections locales de sa formation politique, son convoi fut attaqué, le 5 mai à Soudou, au nord-Togo. L'opposant fut grièvement blessé tandis que d'autres membres du convoi, comme Marc Atidépé, y laissèrent leur vie. Le journal voit derrière cet acte, les « mains noires » du général Eyadéma qui, considérant Gilchrist Olympio comme le rival le plus sérieux qui puisse lui faire ombrage lors des élections présidentielles à venir, aurait demandé à ses militaires de lui faire ce coup. ${ }^{31}$ Pour preuve, le journal évoque le festin organisé au siège du RPT le 5 mai au soir au lendemain 6 mai, pendant que l'opposition et une bonne partie de 
la population était en train de pleurer les morts de Soudou et s'interrogeaient sur le sort de l'opposant Olympio suite à ce guet-apens. ${ }^{32}$

Le même organe relata l'assassinat de l'homme politique Tavio Amorin. Celui-ci a été criblé de balles la nuit du jeudi 23 juillet 1992 dans le quartier de Tokoin Gbonvié à Lomé. Les auteurs de ce forfait réussirent à s'enfuir, sans prendre soin de protéger leurs cartes professionnelles, abandonnées sur les lieux du forfait. Elles appartiennent à deux gardiens de la paix : Yodolou Boukpessi et Kossi Karéwé. La victime fut transportée d'urgence au Centre hospitalier universitaire (CHU) de Lomé-Tokoin où les médecins s'avouèrent incompétents vis-à-vis du cas. Le lendemain, il fut transféré à l'aéroport international de Lomé, d'où il fut évacué sur la France. Mais lors du transfèrement, il fut poignardé au visage, forfait dont les auteurs sont inconnus, ce qui a compliqué son cas. ${ }^{33}$ Il mourut à Paris le 29 juillet $1992 .{ }^{34}$ On reprochait à cet opposant d'être trop critique vis-à-vis du général Eyadéma et de son parti RPT. Au regard des indices retrouvés sur les lieux, il n'y a point de doute, selon les deux organes, que les auteurs de ce forfait sont issus des FAT. Selon La Parole, la preuve que les commanditaires de ce crime sont du RPT est que « Gege (surnom donné par l'organe à Gnassingbé Eyadéma) a fêté la mort de Tavio » à Kara avec les siens, le jour même où arrivait à Lomé, la dépouille mortelle du défunt. ${ }^{35}$

Les honnêtes citoyens n'étaient pas épargnés. C'est l'exemple d'un jeune homme assassiné par les militaires à Tokoin Cébévito à Lomé, dans la nuit du 8 au 9 novembre. Bien que le corps ait été jeté entre les rails pour faire croire qu'il aurait été écrasé par un train, les blessures par balles que portait la victime, les traces des chaussures de militaires sur les lieux ainsi que les témoignages des riverains furent des éléments sur lesquels s'est basée La Parole pour attribuer ce forfait aux FAT. ${ }^{36}$

Ce crime peut sembler ne rien avoir avec les violences politiques. Mais comme souligné plus haut, il participe de la stratégie mise en place par les militaires pour dissuader les populations du soutien qu'elles apportaient aux opposants dans leur ambition de chasser du pouvoir le général Eyadéma. Les exemples sont nombreux.

Une analyse pointue des cas d'assassinat ci-dessus évoqués montre un fond de réalité. Car, les faits ont été révélés par plusieurs auteurs ${ }^{37}$ ainsi que d'autres journaux privés de l'époque (Tribune des Démocrates, Forum Hebdo, etc.) et même ceux proches du RPT (Le démocrate), même si ce dernier n'est souvent pas d'avis sur l'attribution des forfaits aux FAT. Mais ce qui frappe dans la relation des faits par les deux organes à l'étude, c'est la manière dont les évènements sont décrits.

En général, le ton montre une certaine aigreur contre les auteurs des crimes. Les noms des commanditaires, notamment Gnassingbé Eyadéma, les militaires et le RPT sont tournés en dérision : «Ignare-cinglé », «Eyadémon » ou simplement le « démon », «Abalo national» ou « Abalo de Pya » (Abalo 
désignant en kabiyè « garçon »), pour désigner Eyadéma ; « militarés » ou « multi-tarés » pour militaires (La Parole), «FATalités » pour désigner les FAT, « RPiTeux » pour RPT, etc. (Kpakpa désenchanté).

Ces déformations sont significatives. Choisies à dessein, elles rendent compte du contenu, surtout si la déformation se trouve dans un titre, comme par exemple, "La jubilation du RPiteux » à l'annonce du décès de Tavio Amorin. À travers ce titre, on peut lire en filigrane l'idée selon laquelle « il est pitoyable que des gens fêtent le décès d'un citoyen ». Et ces gens, ne sont autres que les membres du RPT. On peut multiplier les exemples. Mais plus que ces déformations, les deux journaux ont eu recours à la caricature pour illustrer, soit le cynisme des auteurs de ces actes, soit leur inconscience. Nous allons surtout nous appuyer sur les caricatures de Kpakpa désenchanté, du fait qu'elles sont plus nettes que celles de La Parole. satirique.

L'attentat de Soudou fut l'un des événements illustrés par la presse

\section{Image $n^{\circ} 1$ : Illustration en rapport avec l'attentat de Soudou et l'assassinat d'Olympio père ${ }^{38}$}

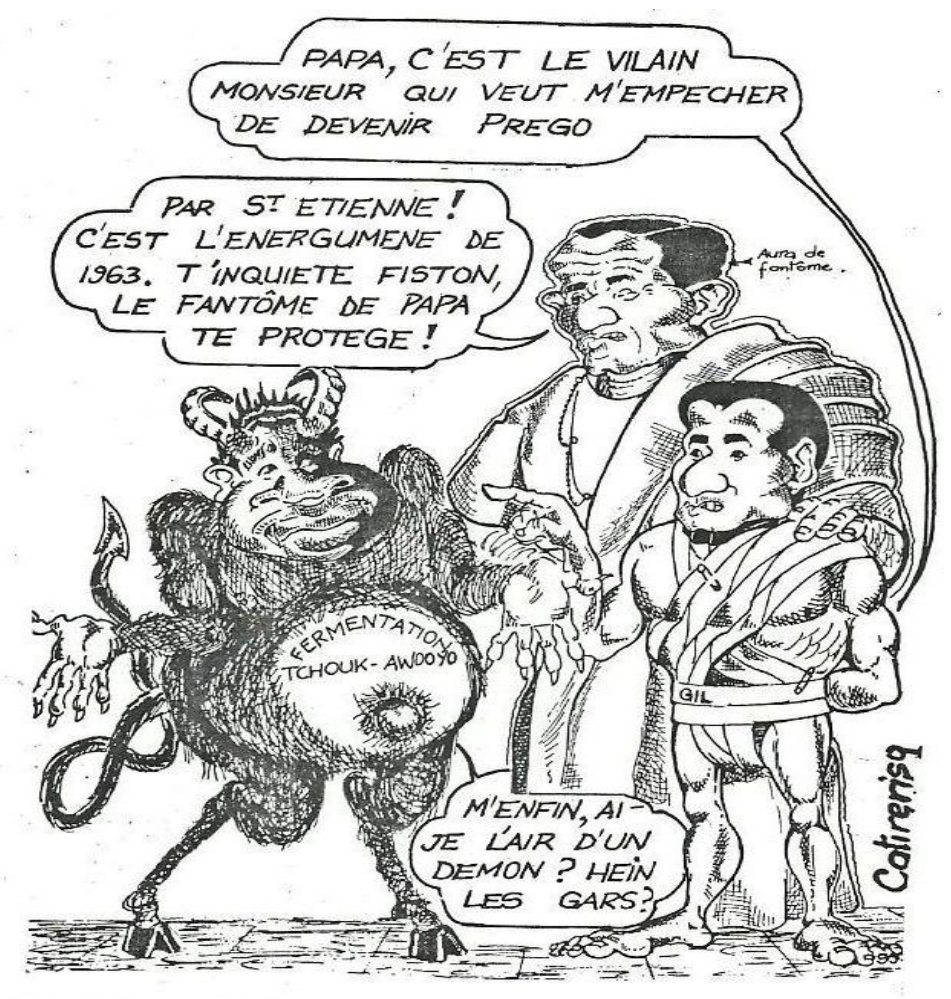

L'illustration faite ici montre Sylvanus Olympio, premier président du Togo accompagné de son fils, Gilchrist Olympio (à sa gauche). Ce dernier 
doigte un animal hideux au visage quelque peu humain qui, selon lui, l'empêche de devenir président du Togo, se référant à l'attentat dont il a été victime à Soudou le 5 mai 1992. Olympio père dévisage l'animal et dit à son fils que c'est l'énergumène de 1963, cause de sa propre mésaventure (faisant allusion au coup d'État dont il a été victime au cours de cette année). Il rassure par la suite son fils, lui disant que son fantôme le protègera contre le démon. Ce dernier réagit, en demandant aux deux s'il avait l'air d'un démon. Selon ce journal, Eyadéma n'est qu'un démon un animal hideux qui, même s'il ressemblait à un homme, ne peut que se saouler la gueule avec les boissons fermentées (Awooyo et Tchoukoutou-bière locale-), comme l'indique l'inscription sur la poitrine de l'animal.

\section{Image $n^{\circ} 2$ : Une autre Illustration en rapport avec l'attentat de Soudou et l'assassinat d'Olympio père ${ }^{39}$}

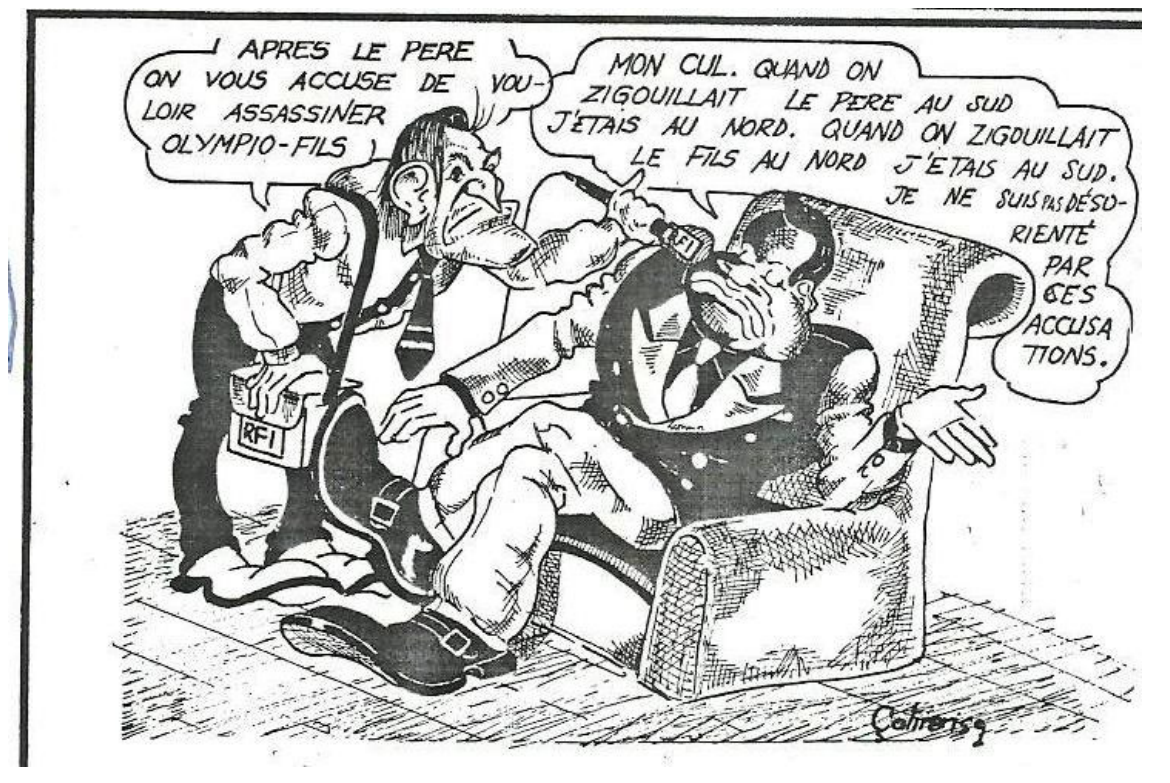

Toujours dans le souci de faire le lien entre les événements de Soudou et ceux ayant entraîné la mort de Sylvanus Olympio en 1963, l'image ci-dessus montre un journaliste de la RFI (apparemment Christophe Bouabouvier), interviewant le président Eyadéma en ces termes : "Après le père, on vous accuse de vouloir assassiner Olympio-fils ». En réponse, Eyadéma déclare : « (...) quand on zigouillait le père au sud, $\mathrm{j}$ 'étais au nord ${ }^{40}$. Quand on zigouillait le fils au nord, j'étais au sud. Je ne suis pas désorienté par ces accusations ».

Ce souci d'inscrire les événements dans le temps et de tenter des rapprochements intéresse à plus d'un titre l'historien, même si par moment, 
le journal donne des informations historiquement erronées. C'est ce souci d'historiciser les violences politiques commises par Eyadéma (ou en son nom) qui transparaît sur l'image suivante.

\section{Image $n^{\circ} 3$ : Les grandes dates de la violence politique au Togo sous Eyadéma $^{41}$}

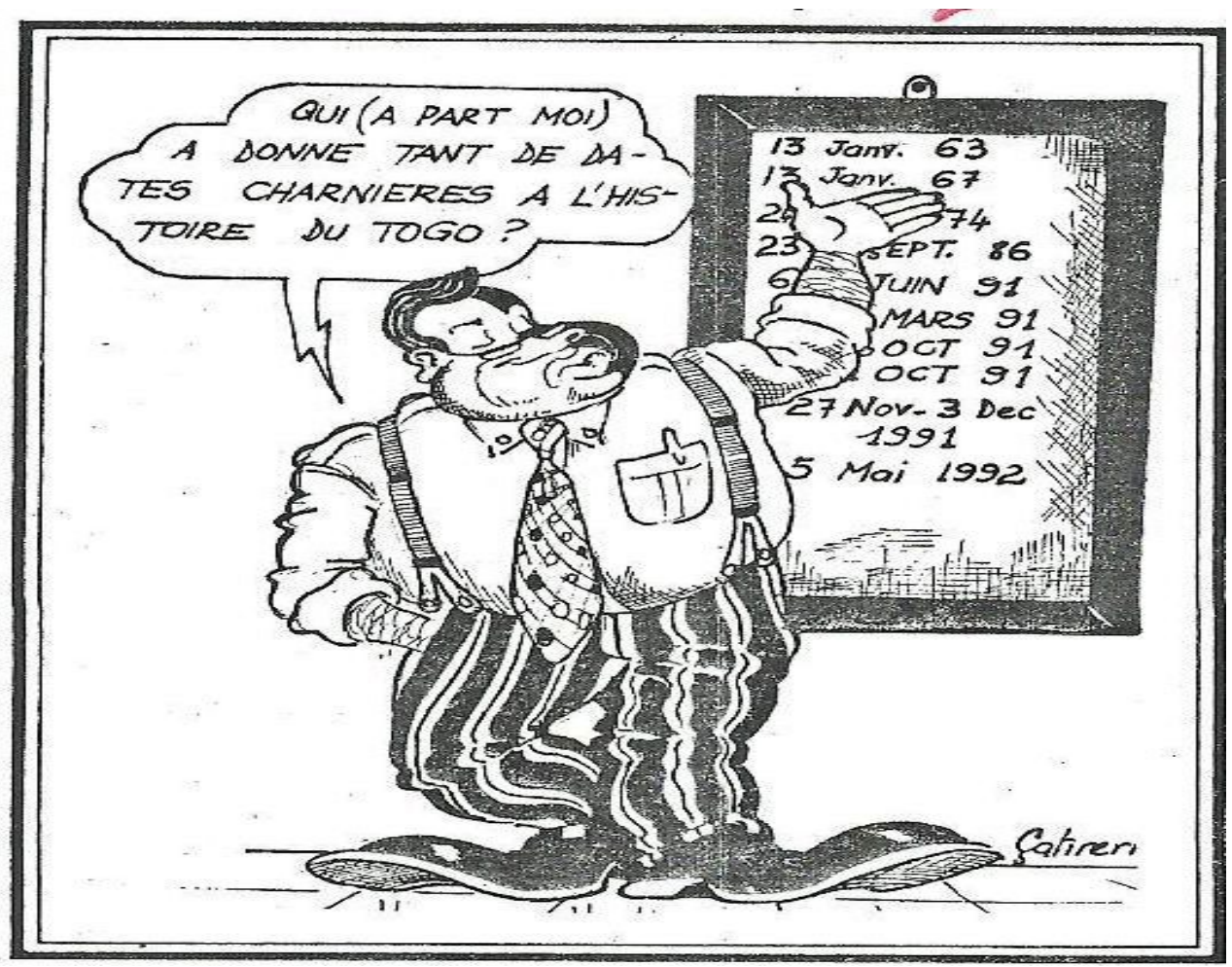

Sur cette image, on voit Eyadéma se vanter des années tragiques connues dans l'histoire du Togo en ces termes : «Qui (à part moi sous-entendu) a donné tant de dates charnières à l'histoire du Togo ? ». On voit que le répertoire des dates part du coup d'État de 1963 précédemment évoqué et s'arrête sur l'attentat de Soudou du 5 mai 1992. À la lecture de cette illustration et du commentaire qui a suivi, on comprend que Kpakpa désenchanté, en parlant de dates charnières, n'évoque pas l'idée de dates ayant servi de transition ou d'articulation entre deux périodes, ce qui n'aurait d'ailleurs aucun sens. Il évoque plutôt l'idée de « charnier » (qu'il accorde maladroitement au féminin), renvoyant à la notion de fosse où sont entassés des cadavres en grand nombre. Eyadema serait alors, à en croire le journal, celui qui serait le champion dans le massacre de nombre de Togolais aux dates indiquées. Même si on peut reprocher une certaine exagération à cette vision des choses, en se référant à l'année 1992 seule, on 
peut postuler que beaucoup ont commis des forfaitures en son nom. C'est le cas de Tavio Amorin, entre autres, qui n'a pas manqué d'inspirer la rédaction de Kpakpa désenchanté.

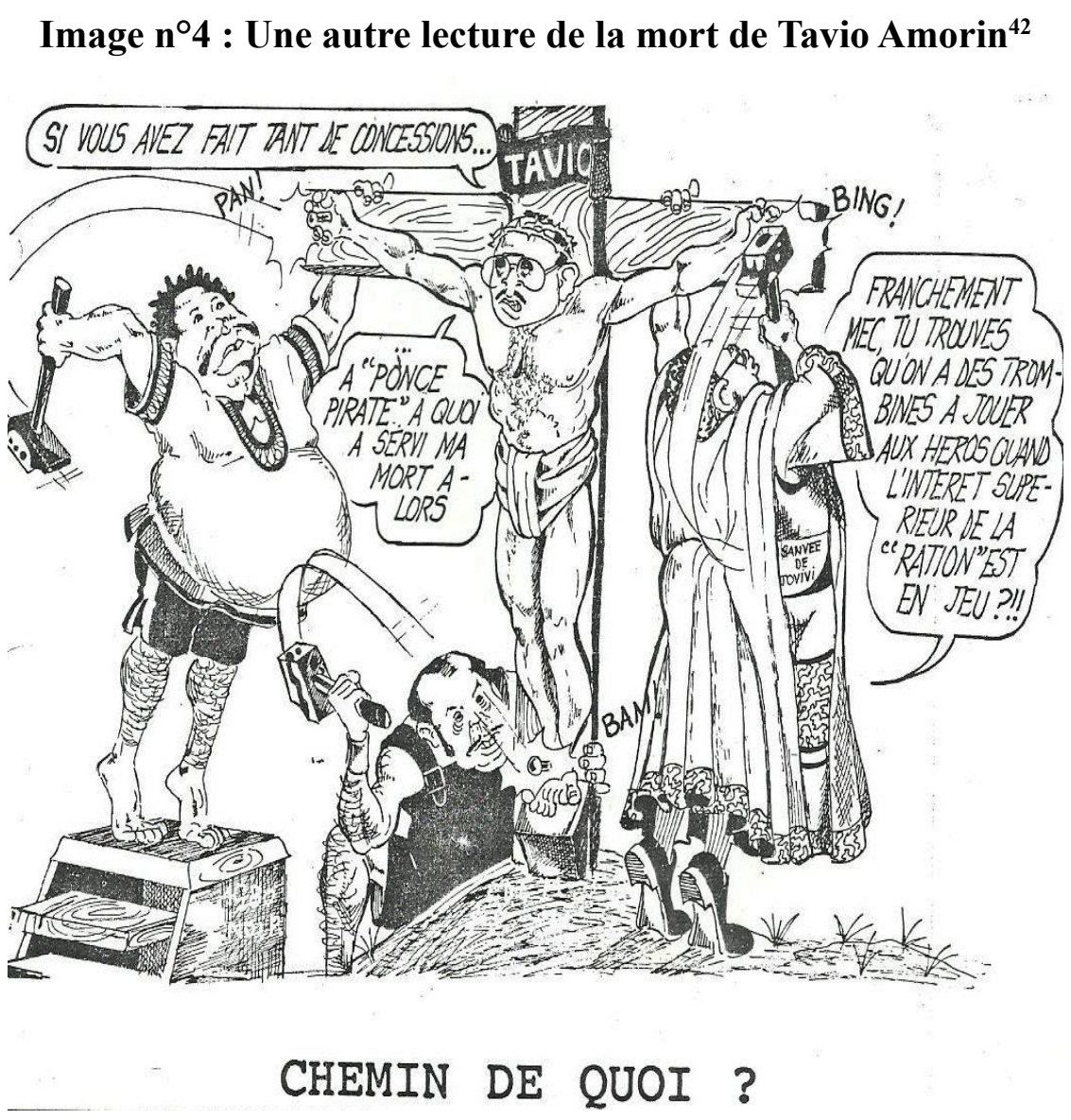

Cette caricature se rapportant à l'assassinat de Tavio Amorin, dont nous avions parlé plus haut, titre « chemin de quoi », tournant en dérision, « le chemin de croix » des catholiques au bout duquel Jésus-Christ a été crucifié. Effectivement, on voit Tavio Amorin en train d'être crucifié. Mais alors, par qui ? Par ses frères de l'opposition tout comme la crucifixion du Christ aussi fut ordonnée par ses frères juifs. Même si la ressemblance est bien forte entre les deux cas comparés ici, il est à rappeler que dans la réalité, Amorin n'a jamais été crucifié. Il a été assassiné par la garde prétorienne. Cela étant, quel est alors le sens réel de cette image ? Le caricaturiste veut simplement montrer que la mort de Tavio Amorin fut finalement une mort gratuite ; car les causes qu'il défendait et qui faisaient de lui un opposant intransigeant, ce qui lui a valu la mort, n'ont pas été véritablement poursuivies par ses frères de l'opposition. 
Voilà pourquoi sur la croix, Amorin pose la question suivante aux opposants : « si vous avez fait tant de concessions à «Ponce pirate», à quoi a servi alors ma mort?». Il fait ainsi allusion à tout ce qui a conduit à remettre le président Eyadema au pouvoir alors qu'Amorin était bien convaincu qu'il quitterait le pouvoir. Koffi Kodjo décrit, à sa manière, ces concessions :

À mesure qu'approchait le terme d'un an fixé pour la fin de la transition, il devenait de plus en plus évident que l'insécurité était téléguidée depuis le palais de Lomé II. La situation était bloquée, les élections impossibles. Il fallait négocier. Fin août 1992, les leaders démocrates durent accepter, en échange de la prolongation de la transition, un nouveau gouvernement où, cette fois, les ministres RPT détenaient l'essentiel des pouvoirs. ${ }^{43}$

Ces quelques exemples montrent à suffisance que la presse satirique n'a pas que recouru au discours imagé pour illustrer les massacres politiques. Elle a souvent appuyé l'écriture par la caricature pour faire sens à son argumentaire. Mais quel crédit peut-on accorder aux informations véhiculées par cette presse?

\subsubsection{Quel crédit l'historien doit-il accorder aux informations} véhiculées par les deux journaux?

Il est clair qu'à la lecture des informations véhiculées par les deux journaux, l'historien a une masse d'informations sur ce qu'ont été les assassinats politiques au cours de l'année 1992, même si tous les cas n'ont pu être cités ni répertoriés dans les colonnes desdits journaux. Mais à l'analyse, il est apparu que pour parvenir au discours scientifique, il a été nécessaire de démêler l'écheveau en faisant la part entre l'information réelle véhiculée et l'exagération qui est le propre de la caricature. Cela relance tout le débat sur la méthodologie à adopter pour tirer matière à histoire des images en général et des caricatures en particulier.

Ce débat a été, à notre avis, tranché par Annie Duprat dans l'ouvrage collectif intitulé Quelle est la place des images en histoire ?, dirigé par Delporte, Gervereau et Maréchal et paru en $2008^{44}$. Selon ces auteurs, pour faire de l'image une source pour écrire l'histoire, il faut procéder par ce qu'ils appellent une « enquête iconographique ». Celle-ci peut être réalisée en passant par trois étapes. La première consiste à s'interroger sur l'objet à étudier ; la deuxième doit amener l'auteur à rechercher les différents éléments iconographiques relatifs à l'objet d'étude ; la troisième doit consister à faire l'étude sérielle et comparative des éléments iconographiques rassemblés en constituant un corpus. Cette démarche doit être rigoureusement adoptée. Et comme l'histoire recommande de diversifier les sources dans la relation des faits, d'autres sources peuvent être convoquées pour servir d'appoint. Elles 
pourront permettre d'avoir des informations pertinentes pouvant faciliter, dans le cas de l'utilisation de la caricature notamment, le travail de critique du discours accompagnant les images. Mis à part la disponibilité des sources, l'historien doit avoir une bonne connaissance du contexte historique de la période à l'étude au risque de biaiser l'analyse.

\section{Conclusion}

Au terme de la présente analyse, il est évident que La parole et Kpakpa désenchanté sont deux journaux satiriques. Même s'ils n'ont pas connu la même durée de vie, ils ont en commun le fait d'avoir eu à dépeindre la situation des violences politiques, particulièrement, les assassinats politiques que le Togo a connus en 1992, après la conférence nationale dont les résultats sont considérés aujourd'hui comme mitigés. Mêlant humour et exagération, ces deux journaux ont permis de comprendre l'état d'esprit qui prévalait au Togo pendant la période étudiée ainsi que les réalités y afférentes.

Même si, pour l'historien, les informations véhiculées sont à prendre avec précaution, ces journaux ont le mérite de donner l'information brute pouvant permettre à l'historien de chercher des sources complémentaires afin de produire un document scientifique.

Dans un contexte où les archives de la période postcoloniale ne sont pas systématiquement conservées, l'historien togolais est obligé de composer avec ces sources primaires au risque de ne pouvoir écrire l'histoire du Togo de cette période. Toutefois, comme le lui impose sa science, l'historien est obligé de croiser ces sources avec d'autres, en utilisant son arme, la critique historienne.

\section{Notes}

\footnotetext{
* Universidade de Lomé. Lomé, Togo. E-mail: jotsigbe@gmail.com

1 TSIGBE, Koffi Nutefé. L'histoire du Togo postcolonial face à l'absence des archives : quelle solution par les images ? Mosaïque, Lomé, $\mathrm{n}^{\circ}$ 15, p. 83-96, 2014.
}

2 DUPRAT, Annie. Histoire et image. In : DELACROIX, Christian ; DOSSE, François ; GARCIA, Patrick et OFFENSTADTT, Nicolas (sous la dir. de). Historiographies, I. Concepts et débats. Paris : Gallimard, 2010, p. 313.

3 Cf. FRUGONI, Chiara. L’histoire par l'image. Médiévales, Paris, $\mathrm{n}^{\circ}$ 22-23, p. 5-12, 1992. ; DUPRAT, Annie. Image et histoire. Outils et méthodes d'analyse des documents iconographiques. Paris : Belin, 2007 ; etc.

4 Lire par exemple DUPRAT, Annie, Histoire de France par la caricature.Paris : Larousse, 1999 ; ou encore RENAULT, Jean-Marie. Censure et caricatures : les images interdites de l'histoire de la presse en France et dans le monde. Montpellier : Pat à Pan, 2006.

5 i) MARGUERAT, Yves et ROUX, Lucien. Trésors cachés du vieux Lomé, l'architecture populaire ancienne de la capitale du Togo. Lomé : Haho, 1993; ii) DAVID, Philippe. Hommage à Alex Acolatsé, l'un des premiers photographes togolais (1890-1975). Lomé : Haho, 
1993; iii) LAUBER, Wolfgang (sous la dir. de). L'Architecture allemande au Togo. Lomé : Haho, 1993; iv) MARGUERAT, Yves. Lomé, fille du commerce. Une brève histoire de la capitale du Togo. Lomé : Presses de l'Université du Bénin, 1996 ; v) MARGUERAT, Yves. L'architecture française et l'œuvre de Georges Coustère au Togo. Paris et Lomé : Haho et Karthala, 2000 ; vi) KLEIN, Bernard. Sokodé, un siècle d'images. Paris/Lomé : Karthala/ Haho, 2001.

6 BATCHANA, Essohanam. Presse et pouvoirs publics au Togo : 1946 à 1998. Université de Lomé : mémoire de DEA en histoire, 2005, p. 66-67.

7 BATCHANA, Essohanam. Op. cit., p. 72.

8 Lire la Une de La Parole ${ }^{\circ} 10$ du 17 avril 1991.

9 Lire la Une de Kpakpa désenchanté, n 13 du 07 janvier 1992.

10 TETE-ADJALOGO, Tété Godwin. Histoire du Togo. La longue nuit de terreur (19632003). Paris : éd. A. J. Presse, 2 vol, 2006.

11 AGBA, Kondi Charles Madjome. Ministre avec Eyadema. Lomé : les éditions de la Rose bleue, 2009.

12 PARTI DES TRAVAILLEURS. Livre noir contre l'impunité au Togo. 13 janvier 1963-13 janvier $2010: 47$ ans d'assassinats et d'impunité au Togo sous la dictature sanglante du clan des Gnassingbé. Lomé : Nyawo, 2011.

13 BATCHANA, Essohanam. La presse privée face à la transition démocratique au Togo (1991-1993). Educom, Revue du Centre d'études et de recherches sur les organisations, la communication et l'éducation (CEROCE), Lomé, n² 2, p. 35-59, 2012.

14 KODJO, Koffi. Violence spontanée et violence politique pour une histoire de la violence urbaine à Lomé, Togo (des origines à 1994). In : KADANGA, Kodjona ; MOUCKAGA, Hugues ; BATCHANA, Essohanam (éds.). Élections et violences politiques en Afrique noire, enjeux et défis cas du Togo après 1990. Lomé : Presses de l'IRES-RDEC, 2014, p. 101-123.

15 AGBA, Kondi Charles Madjome. Ministre avec Eyadema. Lomé : les éditions de la Rose bleue, 2009, p. 243.

16 Idem, p. 256.

17 Idem, p. 266.

18 PARTI DES TRAVAILLEURS. Livre noir contre l'impunité au Togo. 13 janvier 1963-13 janvier $2010: 47$ ans d'assassinats et d'impunité au Togo sous la dictature sanglante du clan des Gnassingbé. Lomé : Nyawo, 2011, p. 126.

19Kpakpa désenchanté, $\mathrm{n}^{\circ} 13$ du 07 janvier 1992, p.1.

20Kpakpa désenchanté, $\mathrm{n}^{\circ} 13$ du 07 janvier 1992, p.1.

21 Idem, p. 12.

22La Parole, $\mathrm{n}^{\circ} 47 \mathrm{du} 1^{\mathrm{er}}$ janvier 1992, p.3.

23 Ibidem.

24 PARTI DES TRAVAILLEURS. Livre noir contre l'impunité au Togo. 13 janvier 1963-13 janvier 2010 : 47 ans d'assassinats et d'impunité au Togo sous la dictature sanglante du clan des Gnassingbé. Lomé : Nyawo, 2011, p. 126-142.

25 Forces armées togolaises. 
26 Commission nationale des droits de l'Homme.

27Kpakpa désenchanté, $\mathrm{n}^{\circ} 16$ du 04 février 1992, p. 2.

28 Ibidem

29 Idem, p. 4.

30Kpakpa désenchanté, $\mathrm{n}^{\circ} 46$ du $1^{\mathrm{er}}$ septembre 1992, p. 2.

31 Certains journaux de l'opposition à l'instar de Le Temps des démocrates ( ${ }^{\circ} 0000 \mathrm{du} 2 \mathrm{au}$ 8 juin 1992, pp. 1-6), Forum Hebdo (n 94 du 10 juillet 1992, p. 3) avouèrent sans ambages que les criminels sont des militaires et proches d'Eyadéma. Mais Le Démocrate, un journal proche du RPT, dans sa parution $n^{\circ} 020$ du 14 mai 1992, p. 2-4, attribue cet acte à l'opposition.

32Kpakpa désenchanté, $\mathrm{n}^{\circ} 30$ du 12 mai 1992, p. 12.

33 Idem, p. 2.

34La Parole, n`80 du 26 août 1992, p.1.

35Idem, p. 3.

36La Parole, $\mathrm{n}^{\circ} 91$ du 11 novembre 1992, p. 4.

37PARTI DES TRAVAILLEURS. Livre noir contre l'impunité au Togo. 13 janvier 1963-13 janvier $2010: 47$ ans d'assassinats et d'impunité au Togo sous la dictature sanglante du clan des Gnassingbé. Lomé : Nyawo, 2011 ; BATCHANA, Essohanam. La presse privée face à la transition démocratique au Togo (1991-1993). Educom, Revue du Centre d'études et de recherches sur les organisations, la communication et l'éducation (CEROCE), Lomé, $\mathrm{n}^{\circ} 2$, p. 35-59, 2012. .

38Source, Kpakpa désenchanté $\mathrm{n}^{\circ} 30$ du 12 mai 1992, p. 1.

39Source, Kpakpa désenchanté n 30 du 12 mai 1992, p. 12.

40 En réalité, Eyadéma n'était pas au nord lors du coup d'Etat de 1963, que d'ailleurs luimême avait revendiqué avec une certaine fierté à sa prise de pouvoir en 1967, avant de se dédire par la suite. Etant donné que jusqu'à aujourd'hui, lumière n'est pas encore faite sur les véritables auteurs de ce coup d'Etat, pour bien des Togolais, c'est Eyadema qui en est responsable, le prenant sur sa propre déclaration. Par ailleurs, même si au moment de l'attentat de Soudou, Eyadema était au sud, le même journal affirme que les auteurs de ce guet-apens ont agi sur ses ordres.

41Source : Kpakpa désenchanté $\mathrm{n}^{\circ} 34$ du 9 juin 1992, p. 3.

42Source : Kpakpa désenchanté $\mathrm{n}^{\circ} 46$ du $1^{\mathrm{er}}$ septembre 1992, p. 1.

43 KODJO, Koffi. Violence spontanée et violence politique pour une histoire de la violence urbaine à Lomé, Togo (des origines à 1994). In : KADANGA, Kodjona ; MOUCKAGA, Hugues ; BATCHANA, Essohanam (éds.). Élections et violences politiques en Afrique noire, enjeux et défis cas du Togo après 1990. Lomé : Presses de l'IRES-RDEC, 2014, p. 120.

44DELPORTE, Christian, GERVEREAU, Laurent et MARECHAL, Denis (sous la dir. de). Quelle est la place des images en histoire? Paris : Nouveau Monde, 2008.

Recebido em: 20/12/2017

Aprovado em: 17/02/2018 